\title{
Implementation of Edge Detection at Multiple Scales
}

\author{
Shekhar Karanwal \\ Computer Science and Engineering Department, \\ Graphic Era Deemed to be University, Dehradun, Uttarakhand, India. \\ Email: shekhar.karanwal@gmail.com
}

Received: 01 December 2020; Accepted: 14 January 2021; Published: 08 February 2021

\begin{abstract}
Edge detection provides a great platform for feature detection which is very useful for applications related to Digital Image Processing and Medical Imaging. Edge detection went through different steps during its life time. There are various operators proposed for edge detection. Some of them are Sobel operator, Prewitt operator, Robert operator, Kirsch operator, Robinson operator, Laplace of Gaussian Operator (LOG) and Canny Operator. Sobel operator, Prewitt operator, Robert operator, Kirsch operator and Robinson operator produces well results in front of quality images but produces bad result in front of noisy images because they have no noise removal filter. For noise removal gaussian filter is mostly used. However Laplace of Gaussian operator and Canny operator use a Gaussian filter for noise removal. The factors which are considered to be most challenging for edge detection are noisy images, direction in which the maximum edges are produced and edge localization. Another factors which are most suitable for finding of appropriate edge detections are Multiscaling and Thresholding. Multiscaling can be done from fine to coarse scale and coarse to fine scale. As far as this paper is concerned this paper provides implementation of edge detection by various edge detection techniques from fine to coarse scale by using Gaussian filter. Different parameter values for Multiscaling and Thresholding were considered and implemented in this paper which is useful for appropriate edge detection. But prior to that we have described various techniques for edge detection. All implementation is performed in MATLAB R2008b using the database of Minear and Parker [7]. The significance of this research is to observe the edges by employing numerous edge detection techniques from fine to coarse scale.
\end{abstract}

Index Terms: Sobel Operator, Prewitt Operator, Gaussian filter, Laplace of Gaussian Operator, Canny Operator, Multiscaling, Thresholding.

\section{Introduction}

Some most challenging tasks in edge detections are edge detection in front of noisy images, direction in which the maximum edges are produced and edge localization. First order derivative operators such as Sobel, Prewitt, Robert, Kirsch and Robinson operators that does not deal with noise factors therefore if there is a noise in images it will be difficult to detect the edges by first order derivative techniques. However second order derivative operators like Laplace of Gaussian operator (LOG) use a noise removal filter known as Gaussian filter for appropriate edge detection. Canny edge detection also uses a Gaussian filter for noise removal. There are various low pass filters [1] available for noise removal. Gaussian filter [2] is one of the most commonly used low pass filter for noise removal. Marr and Hildreth [3] proposed the 2D-Gaussian filter in which image smoothing are obtained at different levels by varying the value of sigma $(\sigma) . \sigma$ is the scaling parameter which takes different values, depending upon the quality of input image. The increasing or decreasing value of $\sigma$ provides edge detection at different levels. But with the decreasing value of $\sigma$, edge appears with increasing noise. The increasing value of $\sigma$ terminates noise but edge may also disappear. Therefore $\sigma$ value should be adjusted properly for appropriate edge detection. Details about Gaussian filter and multiscaling are discussed later. Poobathy et al. [4] provides the comparison of various edge detection operators by utilizing the PSNR and MSE. Dagar et al. [5] provides the comparative analysis of edge detection techniques based on intelligence computing. Singh et al. [6] discovered the implementation based on FPGA for edge detection in color images. Another factor which is useful in edge detection is the edge detection at different directions, because intensity of images are different at different locations, therefore edges are calculated at different directions and the direction which gives the maximum edge will be considered as an appropriate edge detection. Last factor which is very important for edge detection is threshold value to be used for appropriate edge detection. Different threshold values with the scaling factor $\sigma$ were applied on images for proper edge detection. As far as this paper is concerned, this paper explains first order derivative techniques, second order derivative techniques, thresholding and mutiscaling of edges used at various points 
for accurate edge detection with their implementation. The major objective of the research paper is to test the various edge detection techniques at multiple scales. Depending on the amount of scaling the accuracy increases or decreases. Therefore after lots of testing at multiple scales the one which gives the best output will be considered. Section wise paper described as follows: Section 2 describes the first order derivatives techniques, Details about Gaussian filter, Laplace of Gaussian Filter and Canny Edge Detection are described in section 3, Section 4 describes the multiscaling concepts, Section 5 provide implementation and results performed and finally conclusion is drawn on Section 6.

\section{First Order Edge Detection Techniques}

Sobel edge detection [8] is one of the first edge detection techniques which is still used in various applications. Sobel edge detection is also known as gradient based edge detection. Sobel edge detection is used by various researchers in the research field for edge detection. Some of them are described in $[9,10,11]$. The main drawback of Sobel edge detection is that it produces good output in presence of good quality images but produces bad results in front of noisy images. For this work the Sobel Edge detection is implemented for Gray Scale Image and Images which produced after applying Gaussian filter for different values of $\sigma$ on Gray Scale image by using the masks in $\mathrm{x}$ and $\mathrm{y}$ directions, which is then convolved with the Gray Scale image and Gaussian filtered image to produce the gradient images in $\mathrm{x}$ and $\mathrm{y}$ directions. The total gradient image is computed by calculating the square root of addition of square of gradient images in $\mathrm{x}$ and $\mathrm{y}$ directions. Mask used by Sobel is

$$
\left[\begin{array}{lll}
-1 & 0 & 1 \\
-2 & 0 & 2 \\
-1 & 0 & 1
\end{array}\right] \quad\left[\begin{array}{ccc}
1 & 2 & 1 \\
0 & 0 & 0 \\
-1 & -2 & -1
\end{array}\right]
$$

Gradient image at point $(\mathrm{x}, \mathrm{y})$ is given by the formula

$$
\mathrm{G}(\mathrm{x}, \mathrm{y})=\sqrt{\mathrm{G}(\mathrm{x})^{2}+\mathrm{G}(\mathrm{y})^{2}}
$$

and direction formula is given as

$$
\Theta=\tan ^{-}\left(\frac{y}{x}\right)
$$

In another Step a gradient image is calculated in all eight directions and the direction which gives the maximum output will be considered as best possible edge point. Implementation.

Another first order derivative edge detection is the given by Prewitt [11] which is quite similar to Sobel edge detection. The mask used by Prewitt edge detection is shown below.

$$
\left[\begin{array}{lll}
-1 & 0 & 1 \\
-1 & 0 & 1 \\
-1 & 0 & 1
\end{array}\right] \quad\left[\begin{array}{ccc}
1 & 1 & 1 \\
0 & 0 & 0 \\
-1 & -1 & -1
\end{array}\right]
$$

The gradient image is calculated by using the formula

$$
\mathrm{G}(\mathrm{x}, \mathrm{y})=\sqrt{\mathrm{G}(\mathrm{x})^{2}+\mathrm{G}(\mathrm{y})^{2}}
$$

and directional formula is given as

$$
\theta=\tan ^{-}\left(\frac{\mathrm{y}}{\mathrm{x}}\right)
$$

The edges produced between the Sobel edge detection and Prewitt edge detection has a very minor difference due to the mask value used between them. The only difference is in the middle value of masks used for Sobel edge detection and Prewitt edge detection. Image Gradient at point $\mathrm{x}$ and $\mathrm{y}$ is calculated in all eight directions and the direction which gives the maximum output will be considered as most appropriate edge detection.

Another first order derivative edge detection is given by Robert [11]. The mask which is used by Robert is a $2 \times 2$ mask and its values in $\mathrm{x}$ and $\mathrm{y}$ directions are

$$
\left[\begin{array}{cc}
1 & 0 \\
0 & -1
\end{array}\right] \quad\left[\begin{array}{cc}
0 & 1 \\
-1 & 0
\end{array}\right]
$$

The gradient is calculated by formula 


$$
\mathrm{G}(\mathrm{x}, \mathrm{y})=\sqrt{\mathrm{G}(\mathrm{x})^{2}+\mathrm{G}(\mathrm{y})^{2}}
$$

and directional formula is given as

$$
\theta=\tan ^{-}\left(\frac{\mathrm{y}}{\mathrm{x}}\right)
$$

Due to the $2 \times 2$ mask used by Robert, it is not quite effective as Sobel and Prewitt edge techniques. The edges which are produced by Robert edge detection are very less as compared to Sobel and Prewitt edge detection.

Another edge detection technique is given by kirsch [11] whose masks in $\mathrm{x}$ and $\mathrm{y}$ directions are given by

$$
\left[\begin{array}{rrr}
-3 & -3 & 5 \\
-3 & 0 & 5 \\
-3 & -3 & 5
\end{array}\right] \quad\left[\begin{array}{rrr}
5 & 5 & 5 \\
-3 & 0 & -3 \\
-3 & -3 & -3
\end{array}\right]
$$

Gradient image is calculated in all directions by using the gradient and direction formula as in case of Prewitt and Sobel edge detection, and the direction which gives the maximum output will be considered as appropriate edge detection. Another edge detection technique is given by Robinson [11] whose edge mask is given below

$$
\left[\begin{array}{ccc}
-1 & 1 & 1 \\
-1 & -2 & 1 \\
-1 & 1 & 1
\end{array}\right] \quad\left[\begin{array}{ccc}
1 & 1 & 1 \\
1 & -2 & 1 \\
-1 & -1 & -1
\end{array}\right]
$$

Gradient image is calculated in all directions and the direction which gives the maximum output will be considered as appropriate edge detection as in case of other first order derivative operators. But the main drawback of these first order derivatives is they respond well in quality images but not in noisy images.

\section{Gaussian filter, Laplace of Gaussian filter and Canny Edge Detection}

To overcome the noise problem in images, Marr and Hildreth developed his filter known as Gaussian filter [2]. The formula used by Marr and Hildreth for noise removal is given below

$$
g(x, y)=\frac{1}{2 \pi \sigma^{2}} e^{-\left(x^{2}+y^{2} / 2 \sigma^{2}\right)}
$$

Where $\mathrm{x}, \mathrm{y}$ are the coordinates of the image and $\sigma$ is the scaling parameter. By using the various values of sigma, image smoothning is done at different levels. This Gaussian filter works well for low noise image but if there is a large quantity of noise available in image then it will be very difficult to detect edges in images, therefore Laplace operator is used along with the Gaussian filter for Noise Removal and operator is most commonly known as Laplace of Gaussian Operator (LOG). The formula used for the LOG is

$$
\Delta^{2} g(x, y)=\frac{d^{2}}{d x^{2}} g(x, y)+\frac{d^{2}}{d y^{2}} g(x, y)=\frac{x^{2}+y^{2}-2 \sigma^{2}}{2 \pi \sigma^{2}} e^{-\left(x^{2}+y^{2} / 2 \sigma^{2}\right)}
$$

Where $\mathrm{x}, \mathrm{y}$ are the coordinates and $\sigma$ is the scaling parameter. LOG is used to detect edges with noise removal. The scaling parameter of Gaussian filter has gained a huge amount of appreciation in research field. By changing the values of sigma (scaling parameter) edge detection can be done in a more effective way. Different values of $\sigma$ should be tested for extraction of effective edges. Babaud [12] work consists of 1D signal which is smoothed by Gaussian filter and he also shows that when second order derivative is calculated on the 1D signal then zero crossings disappears as scale increases from fine to coarse.

Another operator which is most commonly used throughout the world is given by Canny [13] most commonly known as Canny edge detector. Canny edge detector is the most widely used operator in the history of research field. Canny edge detection focuses on the main prime objectives for edge detection. The three aspects which are most important for edge detection are

1) Proper edge detection.

2) Good Localization.

3) Respond to a proper edge.

Canny proposed a method which consists of all three important features required for edge detection. The major steps of Canny Edge detection are 
1. Canny's method uses a Gaussian filter for noise removal. First step of Canny edge detector is the conversion of Gray scale image to Burred Image by applying the Gaussian filter which was formed by taking the convolution of Gaussian filter with Gray Scale image. The parameters of Gaussian function include size which specifies the size of rows and columns which is to be applied on the gray scale image and scaling factor denoted by sigma. The content of blurring or noise removal depends upon the size and scaling factor used. As the size and scaling factor of Gaussian filter increases the content of blurring becomes more and more and as size and scaling factor decreases content of blurring becomes less.

2. Edge detection is the second step of Canny edge detection which is calculated by first order derivative operators like Sobel or Prewitt operators. Gradient images in $\mathrm{x}$ and $\mathrm{y}$ directions has been calculated by taking the convolution of Gaussian blurred image with Gradients in $\mathrm{x}$ and $\mathrm{y}$ directions. Final gradient image is calculated by taking the square root of summation of gradient images in $\mathrm{x}$ and $\mathrm{y}$ directions.

3. Use of non maximum suppression for proper extraction of edges. Non maximum suppression is also known as edge thinning. Edge thinning is also an important aspect of edge detection, by applying the edge thinning algorithm, the edges becomes clearer and well defined.

4. Canny edge detection uses two thresholds values for edge detection, one for detecting weak edges and one for detecting strong edges. This two threshold factor of Canny becomes very crucial in edge detection. Various thresholds values have been applied on edges and the threshold value which gives the maximum edges will be considered as suitable edge detection.

Another important property of Canny edge detection is the calculation of edges at different scales. Edges can be calculated at different scale values and the scale values which give the maximum edge detection will be considered as most appropriate edge detection. Canny edge detection considered to be most appropriate edge detection for so many years and by today also due to its capability for edge detection. Canny edge detection calculates edges from fine to coarse edge detection which is known as edge synthesis. This fine to coarse edge detection by taking various values of sigma at different scales produces the most efficient edges for edge detection. The concept behind fine to coarse is that after applying a Gaussian filter of size ( $\mathrm{nxm}$ ) with various values of sigma (standard deviation) in increasing order, the content of blurring increases or we can say the amount of noise vanishes out from the image. As far as appropriate edges are concerned, one of the edges which will be considered for final edge detection comes by varying various values of sigma.

\section{Multiscaling for Edge Detection}

Mutiscaling is the most important part for edge detection. Edge detection at multiple scales provides a various options for choosing appropriate edges. Two things are very important in multiscaling whether multiscaling has done form fine to coarse or it has been done from coarse to fine. Fine to coarse means the value of sigma increases with varying size and coarse to fine means the value of sigma decreases with varying size. When moving from fine to coarse, the content of blurring increases with the increasing value of sigma, which is very helpful in noise removal but true edges will also disappears with the increasing value of sigma. However when moving from coarse to fine more true edges will be detected with the decreasing value of sigma, but the content of noise also increases. However as far as the experimental setup is concerned for true edge detection, the values of sigma should be tested at different scales, whether it is fine to coarse or coarse to fine, and the scale which gives the maximum possible outcome will be considered as appropriate edge detection. Huge amount of research has been done on multiscaling. Witkin [14] was among the first ones who work on multiscaling. The concept of Witkins was based on considering the 1D signal and calculating the zero crossings (second derivative) of $1 \mathrm{D}$ signal, $1 \mathrm{D}$ signal is first smoothed by Gaussian function and then edges are derived by varying different values of sigma(standard deviation). This type of representation is known as scale space representation. Bergholm [15] edge detection used to calculate edges from coarse to fine edge detection but prior to this image is smoothed by a Gaussian filter and then edges are detected at different scales. Lacroix [16] edge detection was based on calculating edges from fine to coarse scales using Canny edge detection. Lacroix then uses three scales for their edge detection. Smallest scale gives the finest edge detection because of small value of sigma, largest scale gives the content of blurring due to the increasing value of sigma and the intermediate scale is calculated by the smallest and largest scales. Elder and Zucker [17] study focuses on coarse to fine tracking. They argued that the edge detection at coarse scale depends on the contrast and distance factors and edge detection at fine scale consists of huge amount of noise. Williams and Shah [18] worked on the edge detection at multiple scales, Gaussian filter of different sizes are applied to images for proper edge detection. Schunck [19] uses Gaussian filter for edge detection at multiple scales and its some initial steps are based on Canny edge detection. This section provides the implementation of edge detection at multiple scales. All edge detection operators defined in this paper are implemented from fine to coarse scales. During preprocessing step the Gray Scale Image is convolved with different sizes of Gaussian Filter with various values of sigma to obtain the Blurred image. Then Blurred image is used for edge detection at multiple scales. Edge localization is a prime aspect of any edge detection therefore a great care is taken in the implementation for proper edge detection. 
All working is done on Minear \& Parker database [4] and whole implementation is done on Matlab (R2008b) by using various functions.

\section{Implementation and Results}

For implementation purpose the size of the image taken is 200x250. All implementation of this research paper is performed on MATLAB (R2008b) using the database of Minear and Park [7]. Figure 1 shows three samples of images taken from Minear and Park database. Gaussian filter of different sizes and different scale values were applied to the gray scale images and as size and scale value increases the content of blurring also increases. The size value which was used by all three gray scale images with their corresponding sigma values are [3 3] with sigma=1, [4 4] with sigma=2, [5 5] with sigma=3 and [6 6] with sigma $=4$. Figure 2 shows Sobel edge detection at multiple scales. First part of the figure applies Gaussian filter to Gray scale image of different sizes and different sigma values. In second part Sobel edge is calculated on Gray Scale image and Gaussian filter images. Edges are formed by taking constant threshold value which is .005 and sigma=1, 2, 3 and 4 of sizes [3 3], [4 4], [5 5], [6 6] and as size and sig ma increases noise is reduced but there is some reduction in edges also. In third part edges are formed by increasing both the threshold and sigma values. The thresholds which are used is $.005, .007 .009, .011, .013$ and sigma values are 1, 2, 3 and 4 . Figure 2 shows different phases of edge detection at various threshold and sigma values. It is necessary to choose appropriate threshold value and sigma value for correct edge detection. Figure 3 and Figure 4 shows Prewitt and Robert edge detection by using same parameters as it was used for Sobel edge detection. The difference between these operators is in their mask values used by the operators. The implementation of Sobel, Prewitt and Robert operators were performed on MATLAB (R2008b) by using various functions. For all the implementation MATLAB R2008b is utilized. By utilizing the various functions available in MATLAB. Figure 5 shows Kirsch's operator, during first step Gaussian filter is applied on gray scale images of different size and sigma values for noise removal. Second step is the edge detection at different scales. Different edges are formed at different steps by using sizes of [3 3], [4 4], [5 5], [6 6], appropriate threshold of 100 and sigma values are 1,2,3 and 4. As size and sigma value increases noise becomes low and some true edges also disappear. Figure 6 shows Robinson operator, during first step Gaussian filter is applied on gray scale images of different size and sigma values for noise removal. Second step is the edge detection at different scales. Different edges are formed at different steps by using sizes of [3 3], [4 4], [5 5], [6 6], appropriate threshold of 40 and sigma values are 1, 2, 3 and 4. As size and sigma value increases noise becomes low and some true edges also disappear. Kirsch's and Robinson operators are also implemented in MATLAB (R2008b) and some morphological [20] functions are also used from MATLAB (R2008b) for line detection of Kirsch's and Robinson operators. Figure 7 shows zero crossings of Laplace of Gaussian images at various sigma values. Contrast of the image is improved by applying adaptive histogram equalization [7] function on the image and then zero crossings are calculated by applying different values of sigma in increasing order. As value of sigma increases edges disappears. Figure 8 shows Laplace of Gaussian images by varying threshold values with constant sigma value and varying threshold values with varying sigma values. Firstly Image Contrast is improved by applying adaptive histogram equalized function on Gray Scale Image, secondly parameters which were used are, threshold=.005,.010,.015,.020 with constant sigma value 1 for first part and for second part parameters are, threshold=.005,.010,.015,.020 with sigma values 1,1.2,1.3.1.4. Figure 9 shows Canny edge detection whose parameter consists of two threshold values, sigma value with appropriate size. During first step image contrast is improved by applying adaptive histogram equalization function from MATLAB (R2008b) and secondly constant threshold values which were used for each step is .021 for detecting weak edges and .084 for detecting strong edges with sigma values of .5,1,1.5,2,2.5,3,3.5,4,4.5,5. All implementation of Laplace of Gaussian and Canny edge detection were performed on MATLAB (R2008b) by using functions. Therefore it was clear from all the above implementation that as sigma values increases noise immunity becomes low but true edges becomes disappearing. This is for fine to coarse implementation. But as sigma value decreases more edges appears but noise immunity goes up. In our implementation we have applied various techniques for edge detection at various scale values. But it is up to the researcher to take appropriate values for edge detection. 


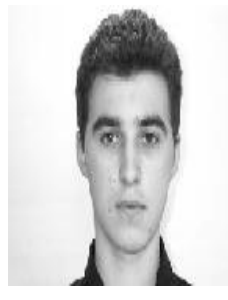

Gray Scale Image

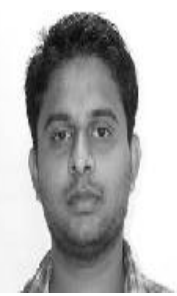

Gray Scale Image

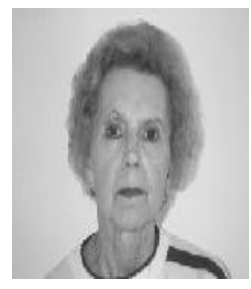

Gray Scale Image

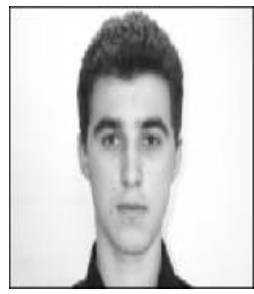

sigma $=1$

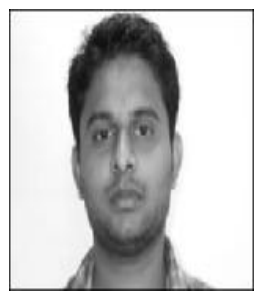

sigma $=1$

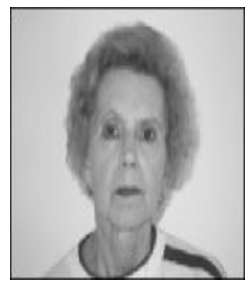

sigma $=1$

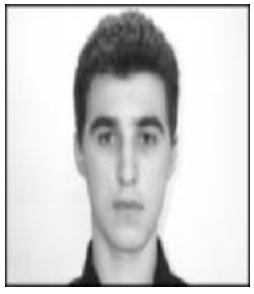

sigma $=2$

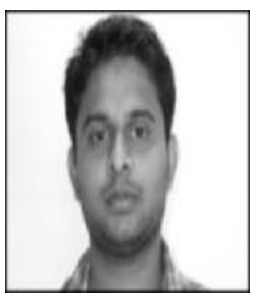

sigma $=2$

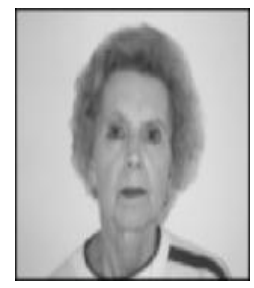

sigma $=2$

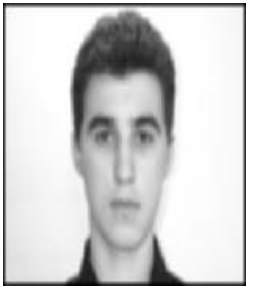

sigma $=3$

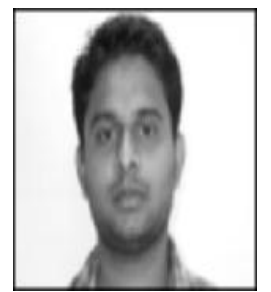

sigma $=3$

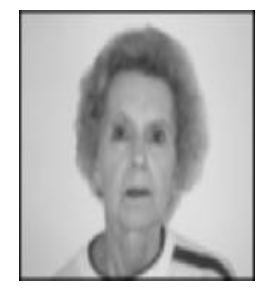

sigma $=3$

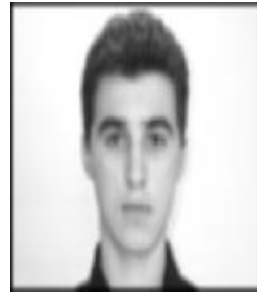

sigma $=4$

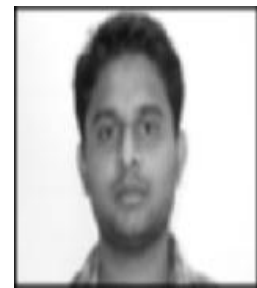

sigma $=4$

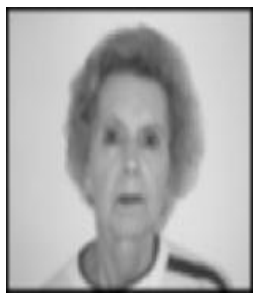

sigma $=4$

Fig. 1. Showing three images and their Gaussian Images with different sizes and different sigma values.
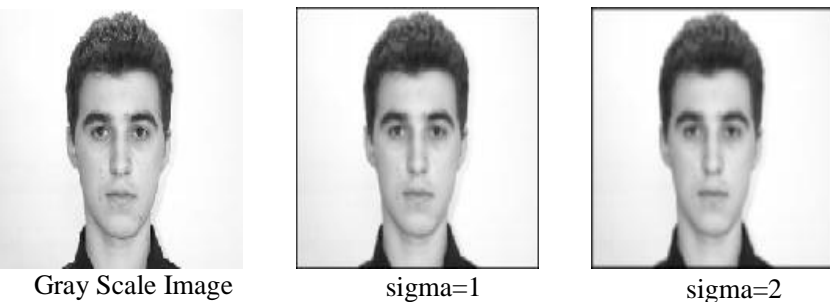

sigma $=2$
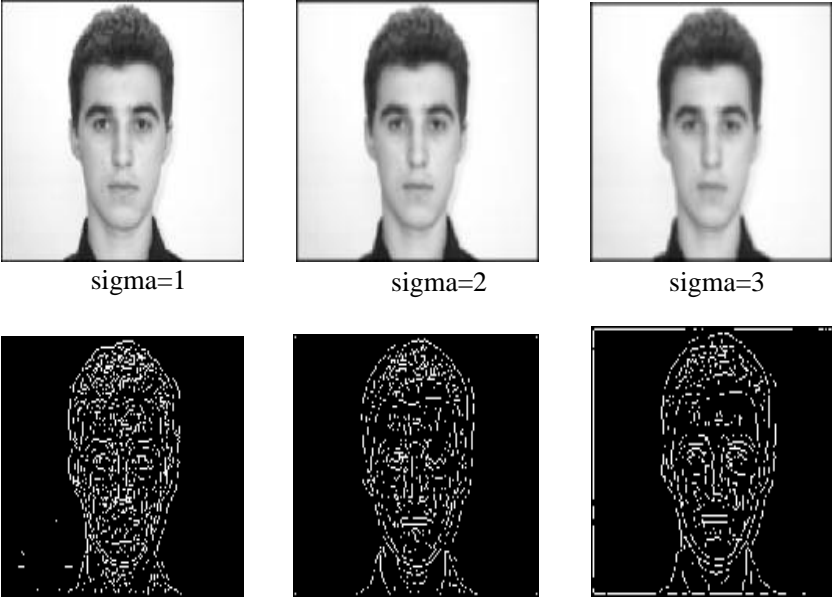

sigma $=3$

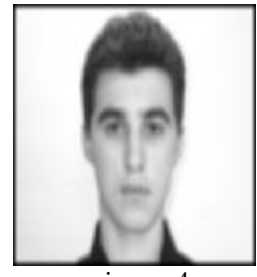

sigma $=4$
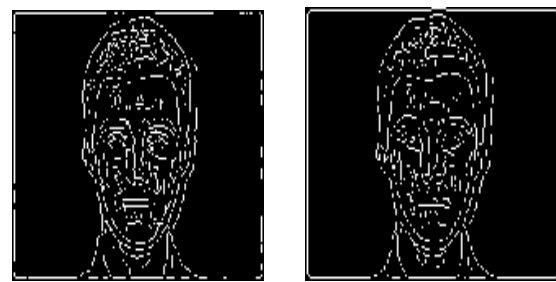

sobel edge with thresh=.005 sobel edge with thresh=.005 Sobel edge with thresh=.005 sobel edge with thresh=.005 sobel edge with thresh=.005
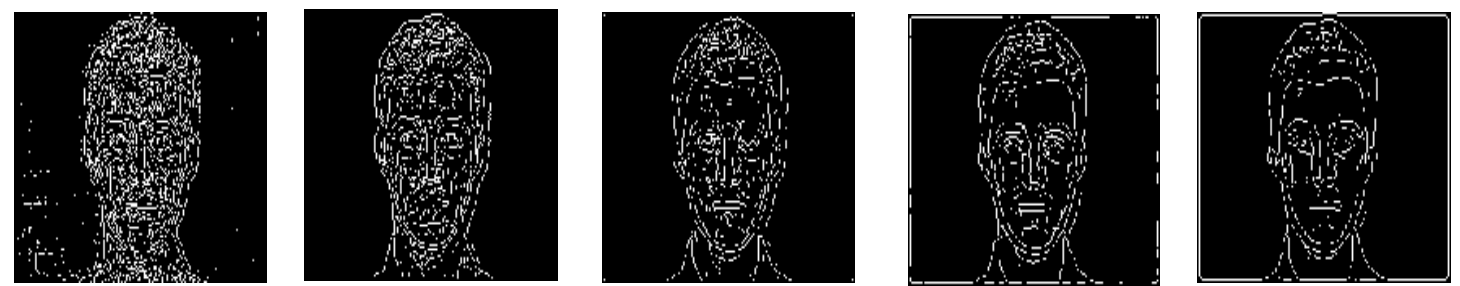

sobel edge with thresh=.005 sobel edge with thresh=.007 sobel edge with thresh=.009 sobel edge with thresh=.011 sobel edge with thresh=.013

Fig. 2. First row of the figure consists of Gaussian Images of neighborhood sizes with varying sigma values, second row of the figure consists of Sobel edge detection with constant threshold values and third row of the figure consists of Sobel edge detection with different threshold values. 


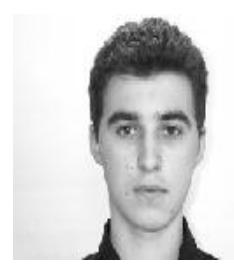

Gray Scale Image

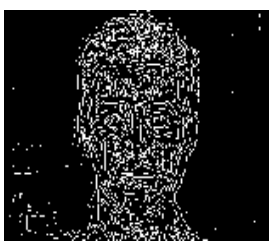

Prewitt edge with th.=.00

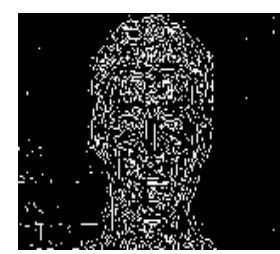

Prewitt edge with th. $=.005$

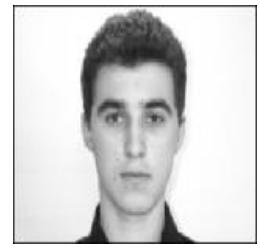

sigma $=1$

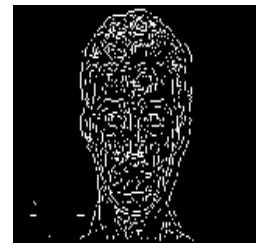

Prewitt edge with th. $=.005$

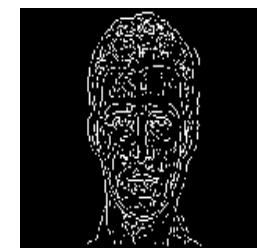

Prewitt edge with th. $=.007$

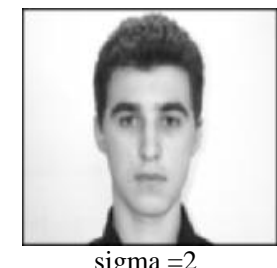

sigma $=2$

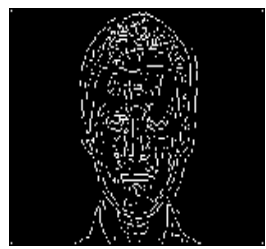

Prewitt edge with th. $=.005$

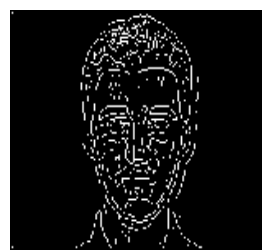

Prewitt edge with th $=009$ Prewitt edge with th $=01$
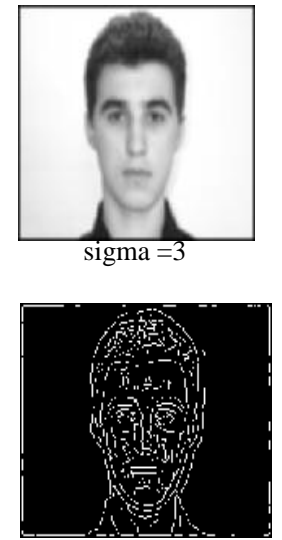

Prewitt edge with th $=00$
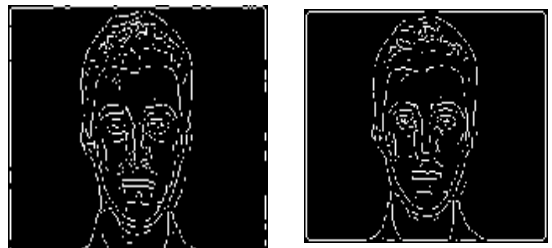

Prewitt edge with th. $=.013$

Fig. 3. First row of the figure consists of Gaussian Images of neighborhood sizes with varying sigma values, second row of the figure consists of Prewitt edge detection with constant threshold values and third row of the figure consists of Prewitt edge detection with different threshold values.

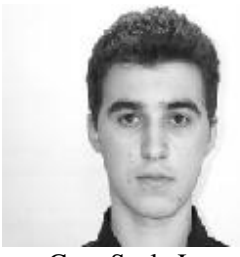

Gray Scale Image

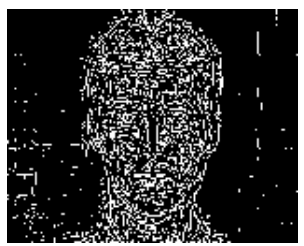

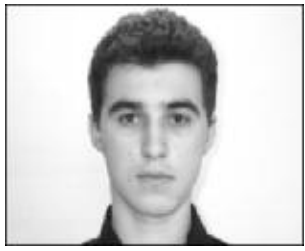

sigma $=1$

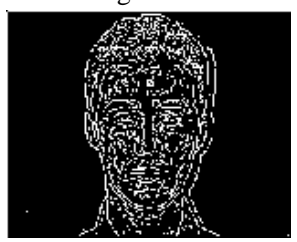

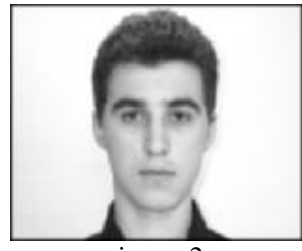

sigma=2

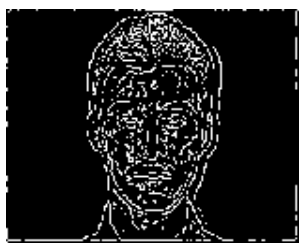

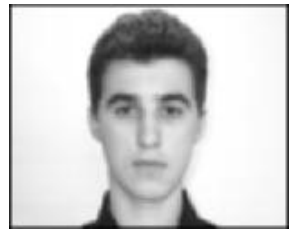

sigma $=3$

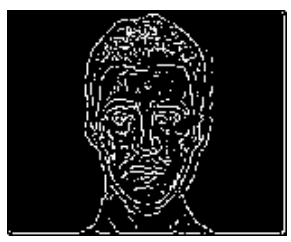

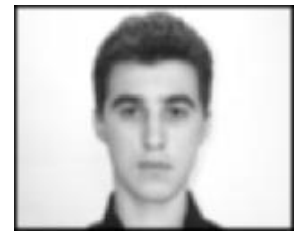

sigma $=4$

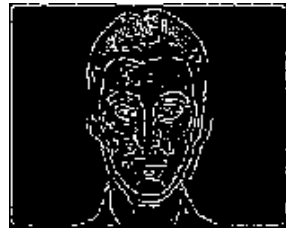

Robert Edge with thresh=.005 Robert Edge with thresh=.005 Robert Edge with thresh=.005Robert Edge with thresh=.005 Robert Edge with thresh=.005
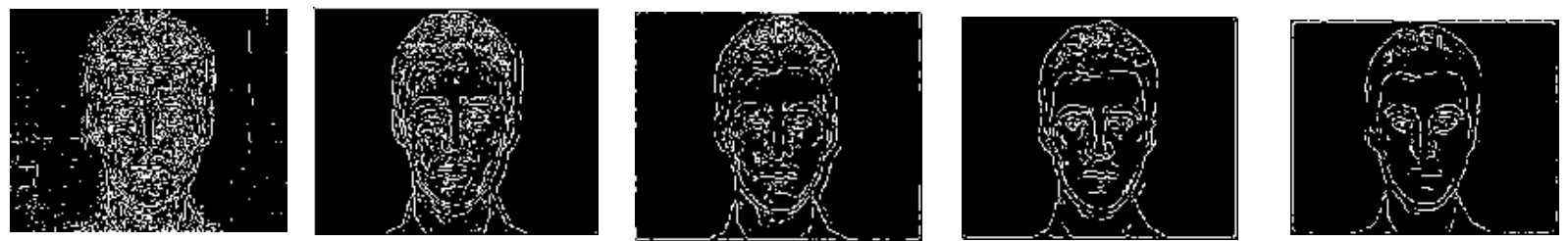

Robert Edge with thresh=.005 Robert Edge with thresh=.007 Robert Edge with thresh=.009 Robert Edge with thresh=.011 Robert Edge with thresh=.013

Fig. 4. First row of the figure consists of Gaussian Images of neighborhood sizes with varying sigma values, second row of the figure consists of Robert edge detection with constant threshold values and third row of the figure consists of Robert edge detection with different threshold values. 


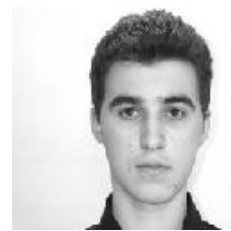

Gray Scale Image

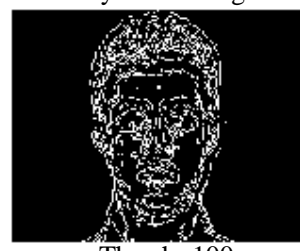

Thresh $=100$
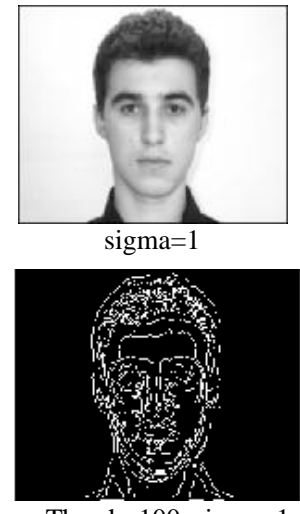

Thresh $=100$, sigma $=1$
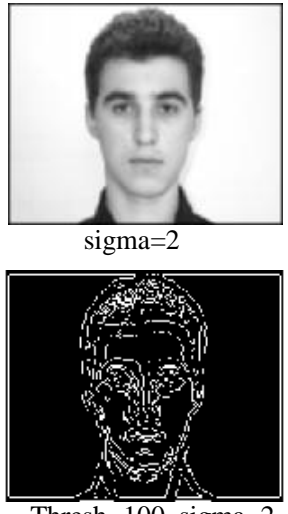

Thresh $=100$, sigma $=2$
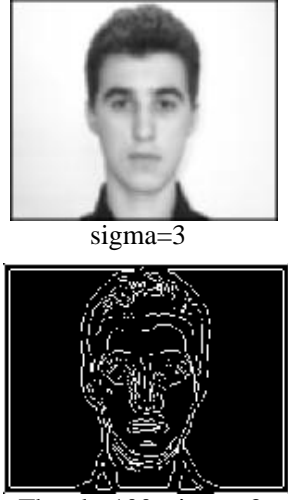

Thresh $=100$, sigma $=3$

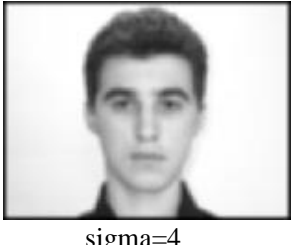

sigma $=4$

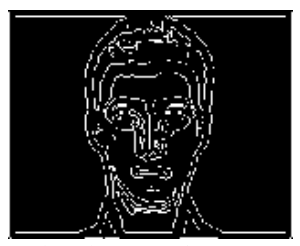

Thresh $=100$, sigma $=4$

Fig. 5. First row of the figure consists of Gaussian Images by varying sigma values and second row of the figure consists of Kirsch's edge detection with their appropriate threshold value and corresponding sigma values.

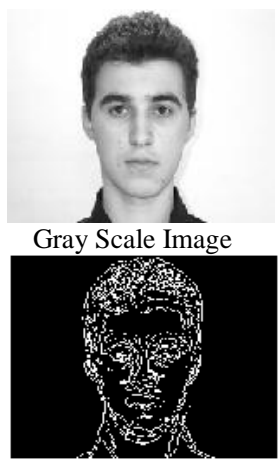

thresh $=40$

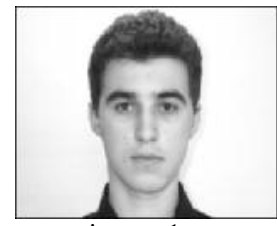

sigma $=1$

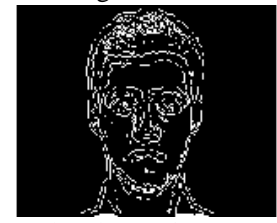

thresh $=40$, sigma $=1$

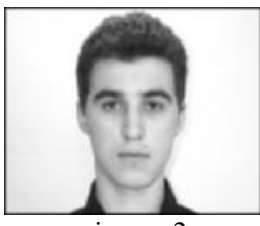

sigma $=2$

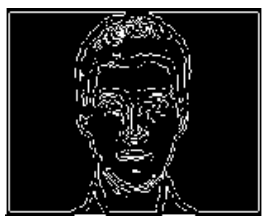

thresh $=40$, sigma $=2$
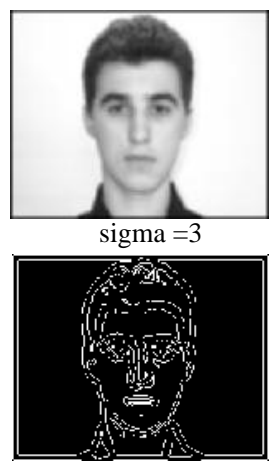

thresh $=40$, sigma $=3$
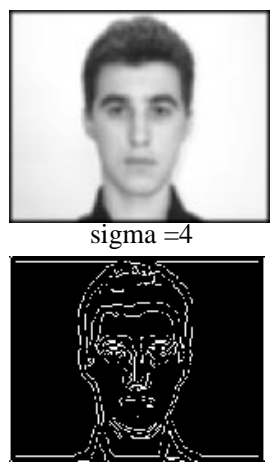

thresh $=40$, sigma $=4$

Fig. 6. First row of the figure shows Gaussian images of different sigma values and second row of the figure shows Robinson edge detection with appropriate threshold values.
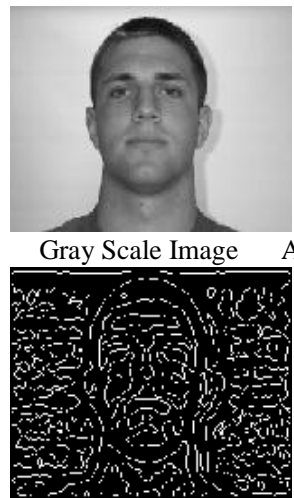

sigma $=3$

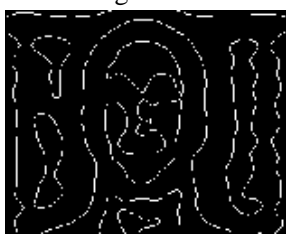

sigma $=8$

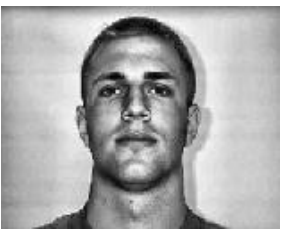

dapt Hist. Equalized Image

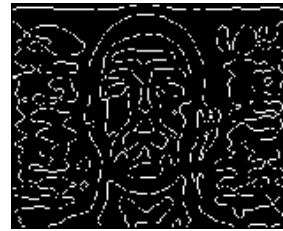

$\operatorname{sigma}=4$

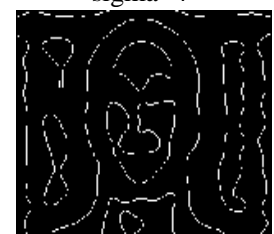

sigma $=9$

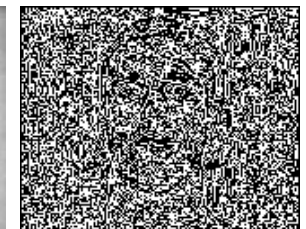

sigma $=.1$

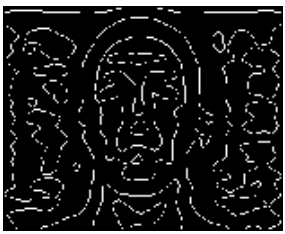

sigma $=5$

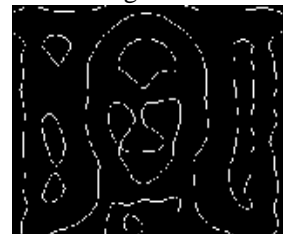

sigma $=10$

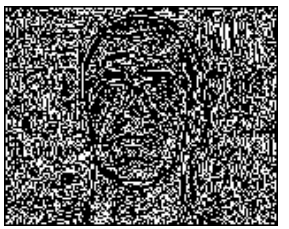

sigma $=1$

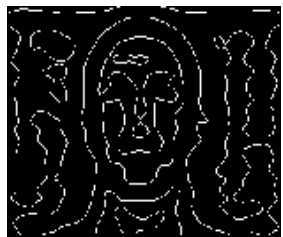

sigma $=6$

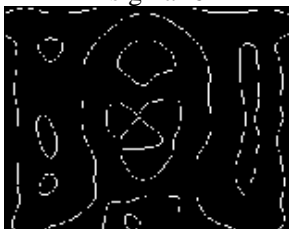

sigma $=11$

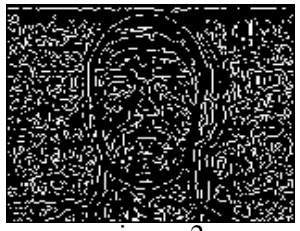

sigma $=2$

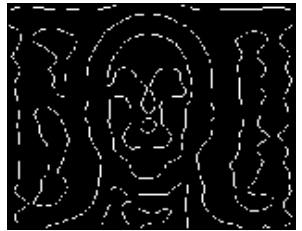

sigma $=7$

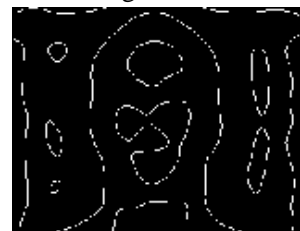

sigma $=12$

Fig. 7. Zero Crossings of Laplace of Gaussian Images for various values of sigma 


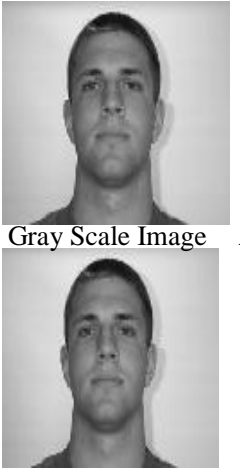

Gray Scale Image

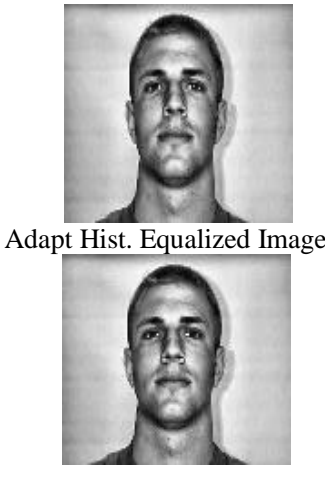

Adapt Hist. Equalized Image

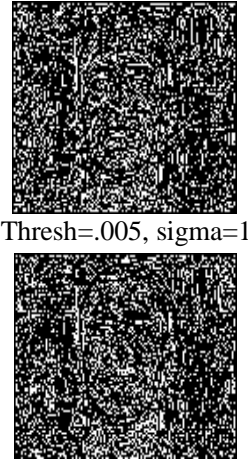

Thresh $=.005$, sigma $=1$

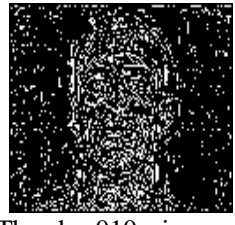

Thresh $=.010$, sigma $=1$

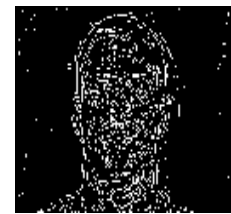

Thresh $=.010$, sigma $=1.2$

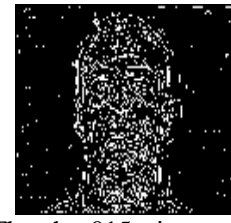

Thresh $=.015$, sigma $=$

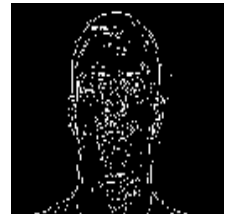

(n)

Fig. 8. First row of figure consists of Laplace of Gaussian Images with varying thresholds values and constant sigma values and second row of the figure consists of Laplace of Gaussian Images with varying thresholds and varying sigma values.

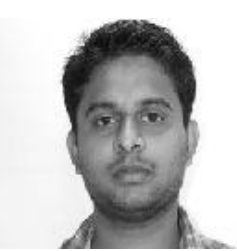

Gray Scale Image

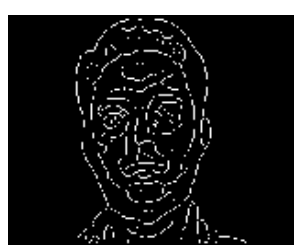

Min Thresh=.021,

Max Thresh=.084,

Sigma $=2$

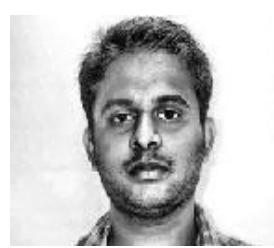

Adaptive Hist. Equalized Image

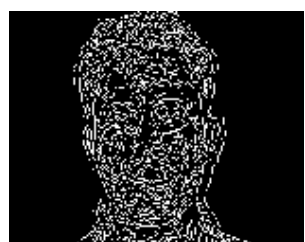

Min Thresh=.021, Max Thresh=.084,

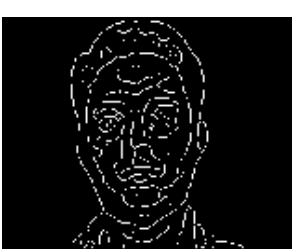

Min Thresh=.021,

Max Thresh=.084, Sigma $=2.5$

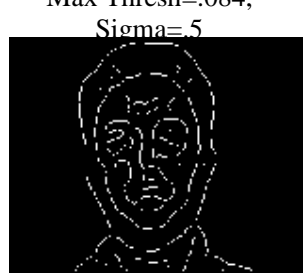

Min Thresh=.021,

Max Thresh=.084,

Sigma $=3$

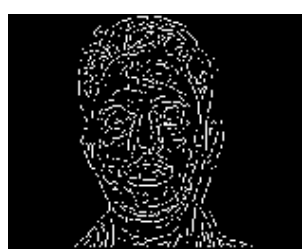

Min Thresh=.021,

Max Thresh=.084,

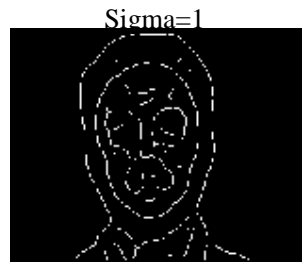

Min Thresh=.021,

Max Thresh=.084,

Sigma $=3.5$

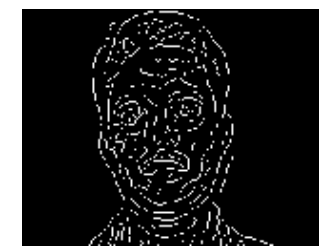

Min Thresh=.021, Max Thresh=.084,

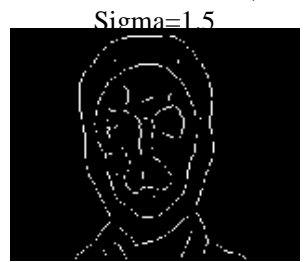

Min Thresh=.021, Max Thresh=.084

Sigma $=4$

Fig. 9. Canny Edge Detection for appropriate threshold values and increasing sigma values

\section{Conclusion}

In this paper the author described and implemented the various techniques of edge detection from fine to coarse by using different scale values and it proves that as the scale value increases noise reduces but true edges may also disappears. However if scale is taken from coarse to fine then more true edges become available but noise immunity also increases. Depending on the type of images available for the processing the proposed concept should be employed. In the next edition the author keen to provide whole application of human recognition system by using all the different phases involve in human recognition system like image enhancement, feature detection, training and classification of data.

\section{References}

[1] R. C. Gonzalez, R.E. Woods. Digital Image Processing, 2nd Ed., Pearson Education Inc. (2002).

[2] M. Basu. "Gaussian based edge detected methods: A survey", IEEE Transactions on systems, man and cybernetics-Part C: Applications and Reviews, 32(3), 2002.

[3] D. Marr and E. Hildreth, "Theory of edge detection", Proc. Royal Society of London, B, 207, 187-217, 1980.

[4] D. Poobathy, R. M. Chezian. "Edge Detection Operators: Peak Signal to Noise Ratio Based Comparison", I.J. Image, Graphics and Signal Processing 10, 55-61, 2014.

[5] N.S. Dagar, P.K. Dahiya, "A Comparative Investigation into Edge Detection Techniques Based on Computational Intelligence", I.J. Image, Graphics and Signal Processing, 7, 58-68, 2019.

[6] S. Singh, A.K. Saini, R. Saini, "Real-time FPGA Based Implementation of Color Image Edge Detection", I.J. Image, Graphics and Signal Processing, 12, 19-25, 2012.

[7] Minear, M. \& Park, D.C. "A lifespan database of adult facial stimuli. Behavior Research Methods, Instruments, \& Computers". 36, 630-633, 2004. 
[8] C. Lopez-Molina, B. De Baets, H. Bustince, J. Sanz, E. Barrenechea, "Multiscale edge detection based on Gaussian Smoothing and Edge Tracking”, Knowledge-Based Systems 44, 101-111, 2013.

[9] M. G. Martini, C. T. Hewage, and B. Villarini, "Image quality assessment based on edge preservation," Signal Processing and Image Communication, 27(8), 875-882, 2012.

[10] W. Gao, L. Yang, X. Zhang, H. Liu, “An improved Sobel edge detection”, In International Conference on Computer Science and Information Technology, 2010.

[11] D. Ziou, S. Tabbone, "Edge Detection Techniques - An Overview". http://citeseer.nj.nec.com/ziou97edge.html, 1997.

[12] J. Babaud, A. P. Witkin, M. Baudin, and R. O. Duda, "Uniqueness of the Gaussian kernel for scale-space filtering," IEEE Transactions on Pattern Analysis and Machine Intelligence, 26-33, 1986.

[13] J. Canny. "A computational approach to edge detection", IEEE Transactions on Pattern Analysis and Machine Intelligence, 8(6):679-698, 1986.

[14] A. P. Witkin "Scale-space filtering", in Proceedings of the International. Joint Conference on Artificial Intelligence, 2, 1983, 1019-1022.

[15] F. Bergholm, "Edge focusing", IEEE Transactions on Pattern Analysis and Machine Intelligence, 726-741, 1987.

[16] V. Lacroix, "The primary raster: A multiresolution image description", in Proceedings of the 10th International Conference on Pattern Recognition, 903-907, 1990.

[17] J. Elder, S. Zucker, "Local scale control for edge detection and blur estimation", IEEE Transactions on Pattern Analysis and Machine Intelligence 20 (7), 699-716, 1998.

[18] D. J. Williams and M. Shah, "Edge contours using multiple scales", Comput. Vis. Graph Image Process., 51, 256-274, 1990.

[19] BG Schunck "Edge detection with Gaussian filters at multiple scales", in Proceedings IEEE Computer Society Workshop on Computer Vision, 208-210, 1987.

[20] G. Papari, P. Campisi, N. Petkov, A. Neri “A biologically motivated multiresolution approach to contour detection”, EURASIP Journal on Advances in Signal Processing, 2007.

\section{Author's Profile}

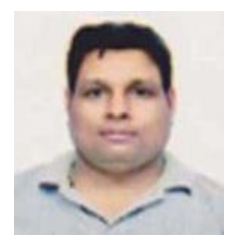

Shekhar Karanwal received his B.Tech. in CS \& IT from IET MJP Rohilkhand University, Bareilly, India. He completed his ME in CSE from PEC University of Technology, Chandigarh, India. He is currently pursuing PhD in CSE from Graphic Era Deemed to be University, Dehradun, Uttarakhand, India. His research interests include Image Processing, Biometrics, Computer vision and pattern recognition.

How to cite this paper: Shekhar Karanwal, " Implementation of Edge Detection at Multiple Scales ", International Journal of Engineering and Manufacturing (IJEM), Vol.11, No.1, pp.1-10, 2021. DOI: 10.5815/ijem.2021.01.01 